\title{
Capture width of the three-float multi-mode multi-resonance broadband wave energy line absorber M4 from laboratory studies with irregular waves of different spectral shape and directional spread
}

\author{
Peter Stansby $^{1}$ - Efrain Carpintero Moreno ${ }^{1}$ - Tim Stallard ${ }^{1}$
}

Received: 13 August 2014 / Accepted: 17 March 2015 / Published online: 2 April 2015

(C) The Author(s) 2015. This article is published with open access at Springerlink.com

\begin{abstract}
A moored multi-body line absorber is an attractive option for offshore wave energy conversion. Laboratory studies have been undertaken to determine capture width with multi-mode excitation and heave resonance for the three-float system M4 where the adjacent float spacing is about half a typical wavelength giving anti-phase forcing. The floats increase in diameter and draft from bow to stern and the bow and mid float are rigidly connected by a beam. A hinge with a damper above the mid float absorbs power from the relative rotation between the bow/mid float and the stern float. The resonant heave frequency for each float is different. Anti-phase surge forcing between mid and stern floats is substantial, while there is no hydrostatic stiffness producing resonance. This represents a hydrodynamically complex system and the laboratory experiments indicate high overall capture widths in irregular waves across a range of peak periods without damping optimisation. With different spectral peakedness and directional spread, the capture width is greater than $20 \%$ of a wavelength (based on the energy period) across a range of peak periods typical of an offshore site for floats with a rounded base. The maximum capture width was about $37 \%$ of a wavelength with rounded base floats; having rounded rather than flat bases increased energy capture by up to $60 \%$ by reducing energy losses due to drag. For floats with flat bases comparisons with a geometrically scaled device five times larger and with similar magnitudes of equivalent damping showed similar capture widths as a proportion of wavelength.
\end{abstract}

Peter Stansby

p.k.stansby@manchester.ac.uk

1 School of Mechanical, Aerospace and Civil Engineering, University of Manchester, Manchester M13 9PL, UK
Keywords Wave energy - Line absorber . Capture width $\cdot$ Multi-mode $\cdot$ Resonance $\cdot$ Irregular waves

\section{Introduction}

Wave energy is a massive renewable energy resource, but effective conversion has proved difficult largely due to its irregular nature. First, we give some general background. Point absorbers are single devices that move in heave, pitch or surge or some combination. Resonance amplifies power generation such that the theoretical maxima in terms of capture width (length of wave crest converted) are $1 / 2 \pi, 1 / \pi$ and $1 / \pi$ wavelengths, respectively, e.g. Falnes (2002). Examples of heaving point absorbers are Archimedes Wave Swing (Polinder et al. 2004) and CETO (Caljouw et al. 2011); examples of pitching devices are PS Frog (McCabe et al. 2006) and Oyster (Cameron et al. 2010). Response to resonance is generally narrow band, although this is normally broader for pitching devices and may be broadened through latching control, e.g. Babarit and Clément (2006). To be effective most devices are designed to function as arrays, either separately tethered to the bed or from a fixed platform, e.g. Manchester Bobber (Lok et al. 2014; Stallard et al. 2009), WaveStar (Hansen and Kramer 2011) or from a floating platform, e.g. Langlee (Lavelle and Kofoed 2011). In another form of point absorption, wave motion is transmitted to an air column, driving oscillatory air motion through a turbine, usually a Wells turbine rotating in one direction, e.g. Masuda et al. (1995) and Ocean energy (2014). Another concept is based on line absorption with Pelamis (Yemm et al. 2012) a well-known example. Here, the device consists of a number of longitudinal cylindrical segments, aligned with the wave direction, connected by hinges at which power is taken off. A segment is typically half a wavelength long, so forcing 
in pitch is maximised. This device is driven principally by buoyancy. The device is floating with a mooring and is usually about two wavelengths long. This has the potential to exceed the capture widths of a single point absorber. A different form of line absorber known as Anaconda (Farley et al. 2012) has the form of a flexible submerged tube designed so that a bulge of water in the tube forms due to the wave pressure and travels at the wave speed, effectively in resonance. There are several good reviews available, e.g. Cruz (2008), Drew et al. (2009), Falcão and de (2010), Falnes (2002), and general hydrodynamic theory for wave-body interaction is developed in Mei (1989).

Some basic principles are apparent. Wave energy resource is greatest offshore which implies relatively deep water deployment (greater than $20 \mathrm{~m}$ say) and hence floating wave energy converters which require mooring. Single devices are of limited value for large-scale generation. Resonance is desirable to optimise power generation, but this is a narrow band process for a single mode and geometry. In terms of engineering practicality, floating moored systems are relatively easy to deploy and maintain relative to fixed supporting structures. Power take-off systems may take various forms with hydraulic systems quite mature, although accessibility above water level is desirable for maintenance.

The present study aims to evaluate a line absorber concept satisfying the following criteria:

1. Automatic alignment with wave direction

2. Forcing in several modes with power contributions combined

3. Multiple resonance with significant power capture over a range of wave frequencies

4. Low damping due to drag and sloshing (due to a body submerging and emerging in water)

5. Single hinge for power take-off (PTO) accessible above deck

6. High capture widths.
An overview of the design process for the system and the range of experimental tests conducted are described in the following section. The experimental results at small scale (geometric scale of approximately 1:40) with devices comprising both flat and rounded base floats are then presented. Experimental results at a five times larger scale with flat bases follow allowing comparison. The results and design are then discussed before conclusions are drawn.

\section{Design}

Three cylindrical vertically axisymmetric floats with circular cross section of different sizes are used with the smallest float for the bow and the largest for the stern as shown in Fig. 1. With the bow float moored, the largest drift force is applied to the stern float and this is beneficial for alignment with the wave direction. Drift force is a second-order inviscid effect, with a mean value in regular waves and slowly varying in irregular waves. For a given float motion, kinetic energy variation and hence average power increase with mass of float. The float diameter should however be less than about $20 \%$ of a wavelength to be in the inertia regime capturing the phase of a wave and negligible diffraction will be desirable for deployment in an array. The stern float should have heave resonance around a prominent wave frequency; this is mainly dependent on draft. The mid and bow float are rigidly connected by a beam to generate a moment relative to the large stern float. This hinge may be above the mid float or at some point between the bow and stern float. Tests have shown that the position above the mid float centreline is desirable and results will only be presented for this case. A single hinge is advantageous in that the PTO will be concentrated in a single hydraulic unit. The mid float should have a draft which gives resonance within the band of prominent wave frequencies; this should be different from that for the stern float to generate

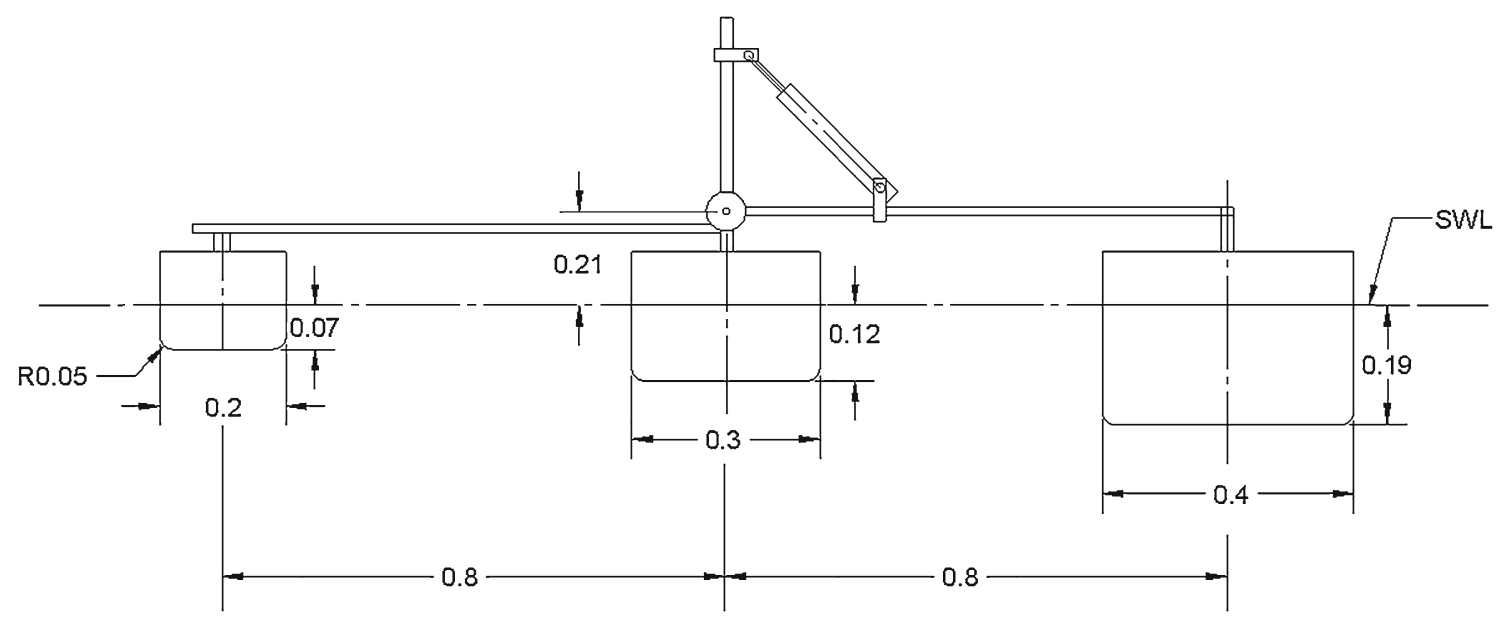

Fig. 1 Diagram of configuration with flat bases and dimensions (m) 
power across the range of wave frequencies for a given site. However, the mid float should not be too big for diffraction to reduce wave action at the stern float reducing its response. The idealised case of two floats acting solely in heave is studied to guide a suitable diameter for mid float. The analytical formulation is shown in the Appendix. A natural period difference between stern and mid float of $20 \%$ is chosen giving drafts in the ratio 1.58:1. Results of this formulation show that maximum power is achieved with corresponding diameters in the ratio 1.33:1. Further optimisation with three floats was not undertaken. Anti-phase heave forcing on the combined bow and mid float will generate pitch. Pitch moment on individual floats will also be generated, but will be relatively small. Surge forces will be substantial, but without a hydrostatic restoring force and at least in a direct linear sense they will not provide a resonant response. The resulting moment about the hinge will be dependent on the hinge position above water level. The moment will be generated by the forces on the combined bow and mid float and the stern float. The force on the bow float will be in anti-phase with the mid float, thus reducing the combined force. The surge force on the bow float should thus be as small as possible to maximise surge moment; this is consistent with what has been already proposed.

Some linearised mathematical modelling has been undertaken in Stansby et al. (2015) for regular waves using diffraction coefficients for heave, pitch and surge from WAMIT (2004) in a time stepping formulation. The floats had flat bases, and imposing drag forces as a function of the velocity of each float with a drag coefficient of about 1.8 gave power output corresponding with the larger-scale experiments (about 1:8 scale). It was shown that removing heave forcing reduced power out by $46 \%$, removing pitch forcing (on individual floats) reduced power by $12 \%$ and, significantly, removing surge forcing reduced power by $57 \%$ for a typical case near resonance. This reinforces the importance of surge forcing and heave resonance and the contribution to power output from different modes being combined. With zero drag coefficient, the maximum capture width was about $75 \%$ of a wavelength which is clearly a high value and may be compared with three floats operating optimally in heave $(48 \%)$ and in surge or pitch (96\%), all hypothetical cases. With further geometry optimisation, the maximum could almost certainly be increased and minimising drag is clearly beneficial. Some experimental results were presented in Stansby et al. (2015). Capture widths were greater than $25 \%$ of a wavelength in regular waves and greater than $20 \%$ of a wavelength in irregular waves across a broad range of wave periods

This paper presents the experimental results for this system obtained with two different base shapes and at two scales in irregular waves. Capture width is considered to be the vital hydrodynamic criterion for the performance. To optimise the geometric configuration preliminary tests were undertaken in regular waves and the effect of relative float spacing, hinge position and bow float size on power have been investigated. Power was then measured with irregular waves with a JONSWAP spectrum with two spectral peakedness factors for unidirectional waves and with two directional spreading factors. Comparisons are made with tests at a five times larger scale with a similar equivalent mechanical damping. Survivability in extreme waves will be considered separately.

\section{Experimental arrangement}

The smaller-scale experiments were undertaken in a wide laboratory flume with $0.45 \mathrm{~m}$ depth, $5 \mathrm{~m}$ width and $18 \mathrm{~m}$ length. Waves are generated by an Edinburgh Designs wavemaker with eight segments enabling regular and irregular waves with directional spreading to be generated using the OCEAN software (Rogers and Bolton King 1997). For each wave condition waves were measured at the device location without the device in position. Wave periods were in the range 0.8 to $1.65 \mathrm{~s}$ and the geometric scale was about 1:40.

Two device configurations were tested. The first has a flat base with slightly rounded corners as already shown in Fig. 1. The stern float diameter is $0.4 \mathrm{~m}$ and the draft $0.19 \mathrm{~m}$. The rounded corners on the base have a radius of $1 \mathrm{~cm}$. The mid float has a diameter of $0.3 \mathrm{~m}$ and a draft of $0.12 \mathrm{~m}$ and the bow float a diameter of $0.2 \mathrm{~m}$ and a draft of $7 \mathrm{~cm}$. The damper is a pneumatic actuator from Norgren Type RM/8016/M/100 connected between the vertical column on the mid float and the connecting beam between the mid and stern float. The hinges comprised simple plastic bearings which will be shown to have very low friction. This is important since small forces were measured. The force on the actuator was measured by a strain-gauged load cell measuring axial force in the range $\pm 20 \mathrm{~N}$, and rotation of the beam about the column was measured by an angular encoder HEDS-9000, code wheel W2000M with 2000 counts per revolution (accurate to $0.18^{\circ}$ ). The force in the load cell is converted to moment about the hinge point and power is simply the actuator force times the piston velocity or equivalently the moment about the hinge times the angular velocity. Ballast is placed in the floats to provide the desired drafts. The mass distribution for each float and components is shown in Table 1 with inertias about the hinge point. This information is also necessary for mathematical modelling. The heave natural periods for each float are shown in Table 2 with added mass from linear diffraction theory provided by WAMIT (2004). The roll period of the combined system acting as a rigid body is close to $1.05 \mathrm{~s}$.

The flat bases were originally chosen because heave forcing is due to oscillating wave pressure on a float base which 
Table 1 Mass distribution and inertias for flat base configuration with origin at the hinge point

\begin{tabular}{llcc}
\hline & Mass $(\mathrm{kg})$ & $x_{G}(\mathrm{~m})$ & $z_{G}(\mathrm{~m})$ \\
\hline Float 1 & 1.85 & -0.8 & -0.2 \\
Beam float 1 to 2 & 0.65 & -0.4 & -0.083 \\
Float 2 & 3.92 & 0.0 & -0.25 \\
Beam float 2 to 3 & 0.475 & 0.4 & 0.0 \\
Float 3 & 4.8 & 0.8 & -0.294 \\
Actuator & 0.196 & 0.16 & 0.16 \\
Ballast float 2 & 4.68 & 0.0 & -0.246 \\
Ballast float 3 & 20 & 0.8 & -0.313 \\
Combined floats 1 and 2 & 11.1 & -0.15 & -0.261 \\
Combined float 3 & 24.8 & 0.8 & -0.30 \\
Inertia about hinge floats 1 and 2 combined & $2.255 \mathrm{~kg} \mathrm{~m}^{2}$ & & \\
Inertia about hinge float 3 & $18.49 \mathrm{~kg} \mathrm{~m}^{2}$ & & \\
\hline
\end{tabular}

Table 2 Heave natural periods for flat base floats based on added mass from WAMIT (2004)

\begin{tabular}{llll}
\hline Float & Diameter $(\mathrm{m})$ & Draft $(\mathrm{m})$ & Heave natural period $(\mathrm{s})$ \\
\hline Bow & 0.2 & 0.07 & 0.698 \\
Mid & 0.3 & 0.12 & 0.88 \\
Stern & 0.4 & 0.19 & 1.10 \\
\hline
\end{tabular}

increases with proximity to the water surface; the flat base enables closest proximity for a given submerged volume. However, drag forces dissipate energy and floats with flat bases have higher drag coefficients than floats with more rounded bases. For a given submerged volume, the draft with more rounded base will be greater and the exciting force less. To investigate their relative significance the mid float was given a hemispherical base of radius $0.15 \mathrm{~m}$ and the stern float was given a rounded corner also of $0.15 \mathrm{~m}$ radius, so that there was a flat central base of $0.1 \mathrm{~m}$ diameter as shown in Fig. 2. The mass distribution for each float and components is shown in Table 3 with inertias about the hinge. The heave natural periods for each float are shown in Table 4 and are only different from the flat base case due to different added mass. The roll period of the combined system was again close to $1.05 \mathrm{~s}$.

\section{Results at smaller scale}

Results are mainly presented as average power generated for different wave height and period with average power converted into capture width as the average power generated divided by the wave power per metre width of crest; this width divided by a wavelength is referred to as capture width ratio. The formula for wave power per metre for regular waves based on linear theory is given by $P=\frac{1}{8} \rho g H^{2} c_{\mathrm{g}} ; \quad c_{\mathrm{g}}=$ $\frac{c}{2}\left(1+\frac{2 k h}{\sinh (2 k h)}\right)$, where $H$ is wave height, $h$ is depth, $k$ is wave number, $c$ is wave velocity and $c_{\mathrm{g}}$ is group velocity, and for irregular waves is given by summation of the power of regular wave components with amplitude defined by a JONSWAP spectrum. To determine the capture width ratio in irregular waves, the wavelength associated with the energy period $T_{\mathrm{e}}$ is most appropriate, equal to $0.84 T_{\mathrm{p}}$ for $\gamma=3.3$ and $0.78 T_{\mathrm{p}}$ for $\gamma=1$ where $T_{\mathrm{p}}$ is the peak period and $\gamma$ is the spectral peakedness factor of the JONSWAP spectrum.

\subsection{Preliminary regular wave tests}

In the smaller-scale experiments, reflections were evident with regular waves making absolute magnitudes difficult to assess. Reflection was relatively low in the larger-scale tests, approximately $6 \%$ without the body present in the basin and are reported elsewhere (Stansby et al. 2015). Regular wave results at smaller scale are thus not presented, although relative power magnitudes were used to assess the geometric configuration. The power with a smaller bow float diameter of $0.16 \mathrm{~m}$ was compared with that due to $0.2 \mathrm{~m}$. Both diameters gave very similar power output overall, but the larger diameter had greater buoyancy to support the connecting beam which would be beneficial at full scale. The effect of reducing the spacing between the bow and mid float was tested and $0.8 \mathrm{~m}$ gave higher power outputs than 0.7 and $0.6 \mathrm{~m}$. The effect of hinge elevations on average power at $0.15,0.21$ and $0.25 \mathrm{~m}$ above mean water level was tested and the $0.21 \mathrm{~m}$ elevation overall gave marginally larger overall power output and was subsequently used.

The variations of average power output with $H^{2}$ showed a closely linear dependence for different periods, which may be expected since wave power is proportional to $H^{2}$. The straight line fits for different wave frequencies passing close to the origin, indicating that power losses due to friction in the bearings are very small. Such small values are difficult to 


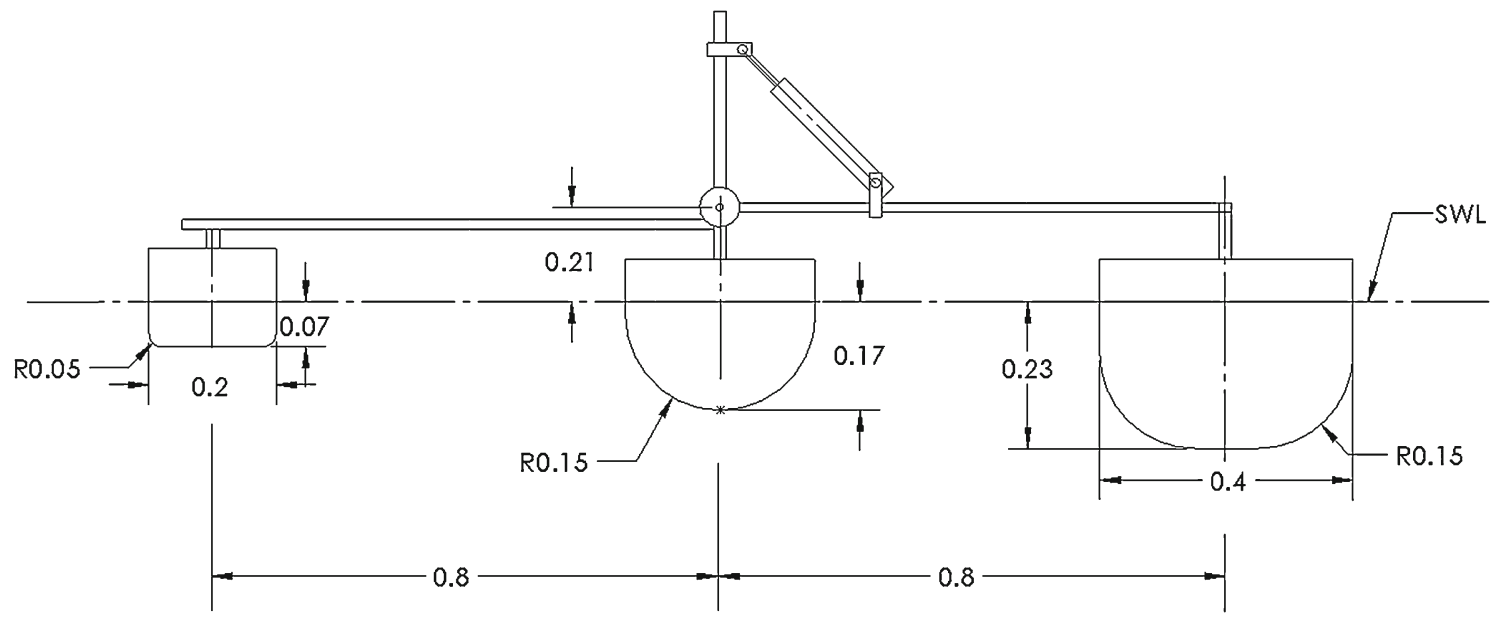

Fig. 2 Sketch of configuration with rounded bases and dimensions (m)

Table 3 Mass distribution and inertias for rounded base configuration with origin at hinge point

\begin{tabular}{llcc}
\hline & Mass $(\mathrm{kg})$ & $x_{\mathrm{G}}(\mathrm{m})$ & $z_{\mathrm{G}}(\mathrm{m})$ \\
\hline Float 1 & 1.85 & -0.8 & -0.2 \\
Beam float 1 to 2 & 0.65 & -0.4 & -0.083 \\
Float 2 & 2.63 & 0.0 & -0.21 \\
Beam float 2 to 3 & 0.48 & 0.4 & -0.0 \\
Float 3 & 5.49 & 0.8 & -0.268 \\
Actuator & 0.19 & 0.16 & 0.16 \\
Ballast float 2 & 6.35 & 0.0 & -0.3 \\
Ballast float 3 & 19.0 & 0.8 & -0.37 \\
Combined floats 1 and 2 & 11.48 & -0.15 & -0.265 \\
Combined float 3 & 24.49 & 0.8 & -0.335 \\
Inertia about hinge floats 1 and 2 combined & $2.28 \mathrm{~kg} \mathrm{~m}^{2}$ & & \\
Inertia about hinge float 3 & $18.81 \mathrm{~kg} \mathrm{~m}^{2}$ & & \\
\hline
\end{tabular}

Table 4 Heave natural heave periods for rounded base floats based on added mass from WAMIT (2004)

\begin{tabular}{llll}
\hline Float & Diameter $(\mathrm{m})$ & Draft $(\mathrm{m})$ & Heave period $(\mathrm{s})$ \\
\hline Bow & 0.2 & 0.07 & 0.698 \\
Mid & 0.3 & 0.17 & 0.82 \\
Stern & 0.4 & 0.23 & 1.02 \\
\hline
\end{tabular}

quantify accurately and will be ignored; the power estimates are thus slightly conservative.

\subsection{Irregular waves}

Capture width ratios (with wavelength defined by $T_{\mathrm{e}}$ ) are shown in Fig. 3 for the rounded base with a flat base comparison for the narrow band JONSWAP spectrum with $\gamma=3.3$. Note the mechanical damping was not adjusted and will be shown to be suboptimal. There is a single peak close to

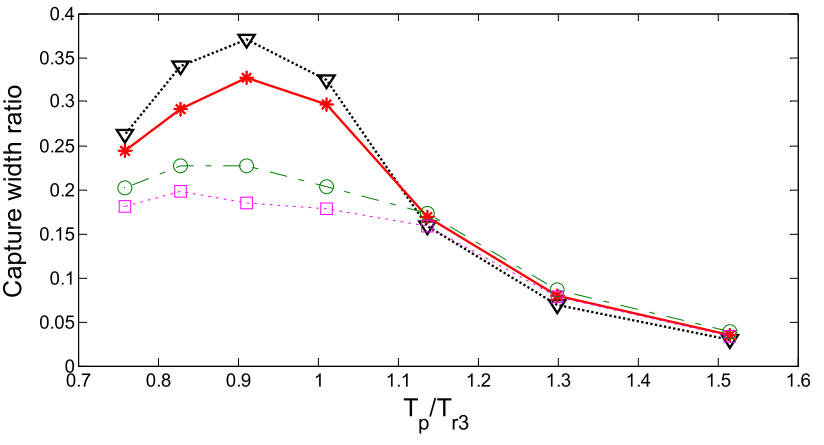

Fig. 3 Variation of capture width ratio (based on $T_{\mathrm{e}}$ ) with ratio of peak period $T_{\mathrm{p}}$ to resonant heave period on stern float $T_{\mathrm{r} 3}$ (based on WAMIT added mass) for uni-directional irregular waves for flat and rounded bases with $\gamma=3.3$. Rounded bases $H_{\mathrm{s}} \approx 0.027 \mathrm{~m}$ (invertedtriangle), $H_{\mathrm{S}} \approx 0.042 \mathrm{~m}$ (asterisk); flat bases $H_{\mathrm{s}} \approx 0.027 \mathrm{~m}$ (open circle), $H_{\mathrm{s}} \approx$ $0.040 \mathrm{~m}$ (open square)

$T_{\mathrm{p}} / T_{\mathrm{r} 3}=0.9$ where $T_{\mathrm{r} 3}$ is the heave resonance period on the stern float with added mass defined by WAMIT (2004) and $T_{\mathrm{p}}=1.0 \mathrm{~s}\left(T_{\mathrm{e}}=0.84 \mathrm{~s}\right)$. The capture width ratios 
are always greater with the rounded bases with a maximum of 0.32 rather than 0.20 with significant wave height $H_{\mathrm{s}} \approx$ $0.04 \mathrm{~m}$, a $60 \%$ increase. Note with $H_{\mathrm{s}} \approx 0.027 \mathrm{~m}$ the maximum ratio is 0.37 , indicating a smaller drag effect with the smaller wave height. The $H_{\mathrm{s}}$ values given in the figure captions are those measured without the device in position and are an approximate value across a frequency range, as the target value was not produced directly. The measured values for each test are used to specify the non-dimensional capture width ratio.

An example of time variation of moment $M_{\mathrm{d}}$, angular displacement $\theta$ and power output is shown in Fig. 4 for $H_{\mathrm{s}}=0.043 \mathrm{~m}, T_{\mathrm{p}}=1.11 \mathrm{~s}$ and $\gamma=3.3$. In a perfect damper, the coefficient $B_{\mathrm{d}}$ would be constant but this was not the case. The $M_{\mathrm{d}}$ time variation is fitted by the least squares method to a model defined as $M_{\mathrm{fit}}=B_{0}+B_{d} \dot{\theta}+B_{a} \ddot{\theta}$, where $B_{\mathrm{a}}$ allows for inertia effects and $B_{0}$ accounts for the small mean value observed. The least squares goodness-of-fit $R^{2}$ of 0.9 is a typical value. The $B_{\mathrm{d}}$ value was $1.65 \mathrm{Nms}$ in this case and was in the range $1.4-2.3 \mathrm{Nms}$ for all tests.

The effect of reducing the spectral peakedness factor $\gamma$ from 3.3 to 1.0 is shown in Fig. 5 for the rounded base floats. The capture width ratio is reduced for the smaller $\gamma$ and is again smaller for the larger $H_{\mathrm{s}}$, although this is a small effect with $\gamma=1$.

The effect of directional spreading is shown in Fig. 6 for $\gamma=3.3$ also for the rounded base floats. The capture width ratio is largest for uni-directional waves $(s=\infty)$ and is reduced with $s=30$ and 5 which are similar. Again, capture width ratio is greater for smaller $H_{\mathrm{s}}$. The directional spreading parameter s defines a spreading function of cosine shape, $\cos ^{\mathrm{S}}(\theta)$, proposed by Borgman (1969) following LonguetHiggins et al. (1963), and is the standard in OCEAN software.
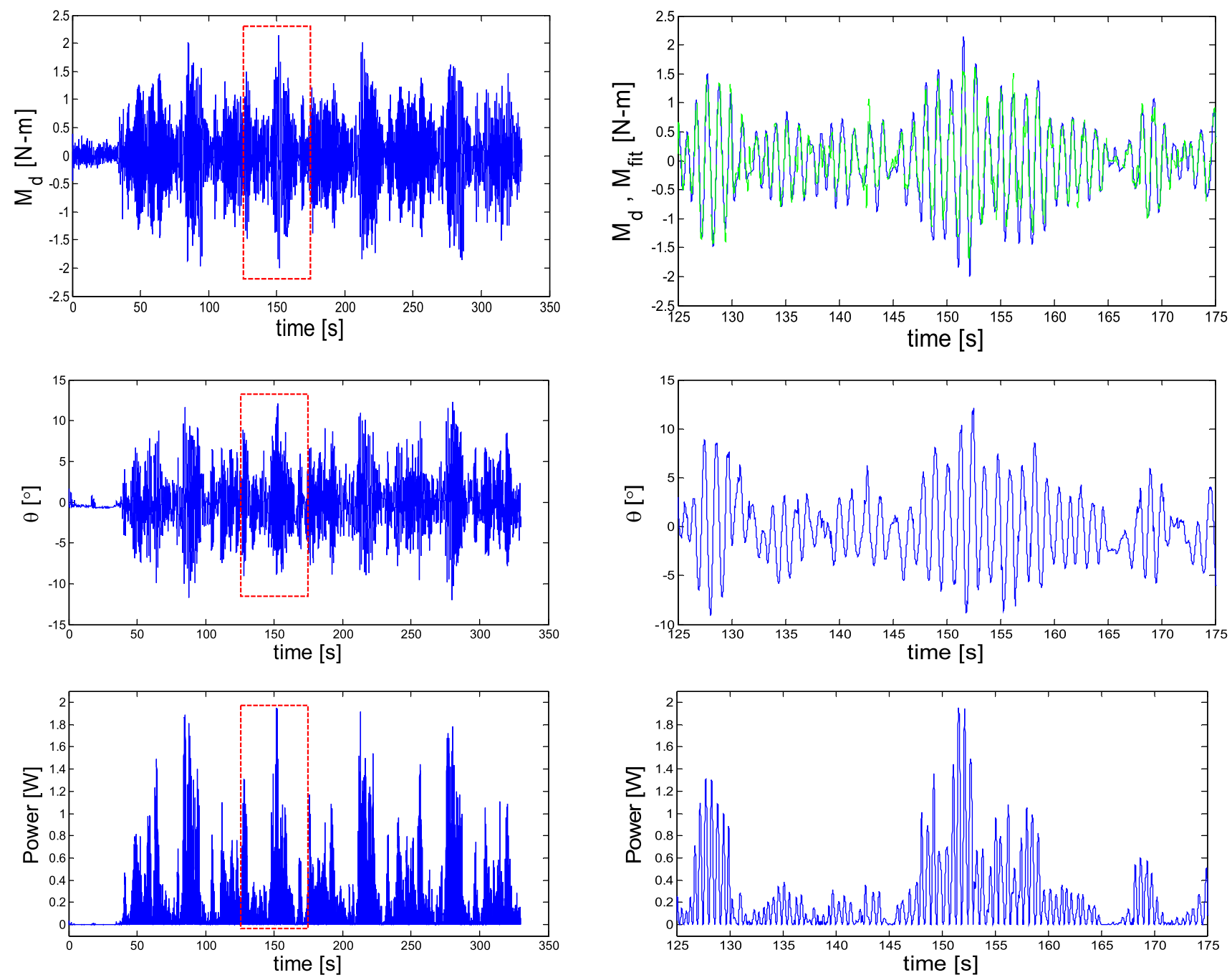

Fig. 4 Variation of moment $M_{\mathrm{d}}$, angle $\theta$ and power output with time for $H_{\mathrm{s}}=0.043 \mathrm{~m}, T_{\mathrm{p}}=1.11 \mathrm{~s}$ and $\gamma=3.3$ with rounded base floats. The right column shows in detail the interval shown in the red box in

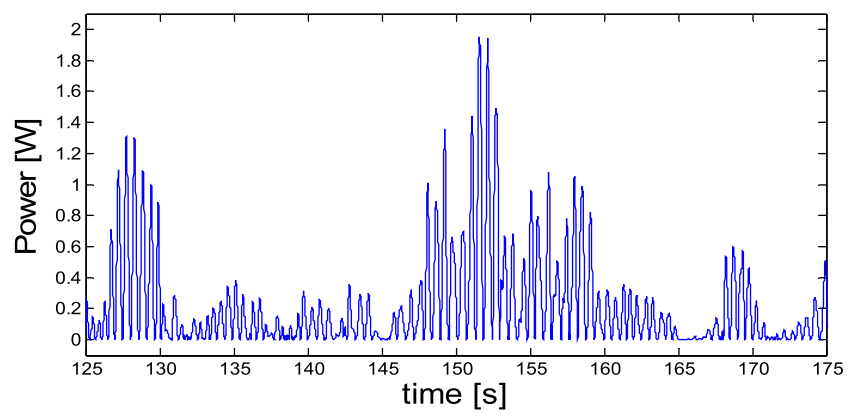

the left column. For $M_{\mathrm{d}}$ the curve fit is shown by the dashed line on the right hand side 


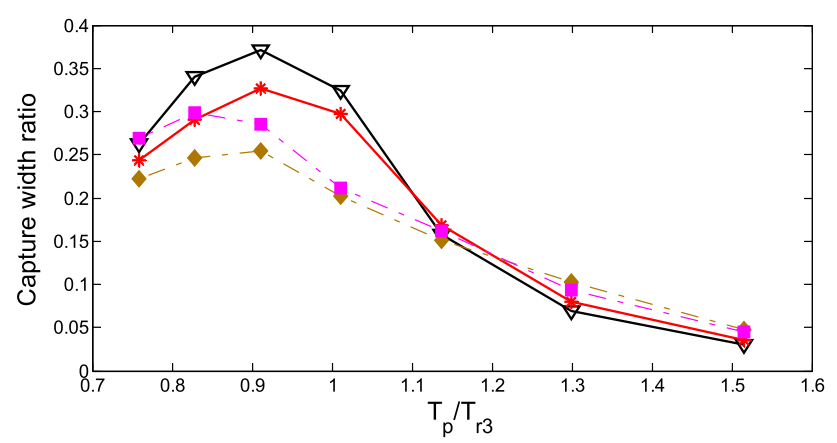

Fig. 5 Variation of capture width ratio (based on $T_{\mathrm{e}}$ ) with ratio of peak period $T_{\mathrm{p}}$ to resonant heave period on stern float $T_{\mathrm{r} 3}$ (based on WAMIT added mass) for uni-directional irregular waves with $\gamma=3.3$ and 1.0 for rounded base floats. $\gamma=3.3 H_{\mathrm{s}} \approx 0.027$ (down triangle), $H_{\mathrm{s}} \approx$ $0.042 \mathrm{~m}$ (asterisk); $\gamma=1.0 H_{\mathrm{s}} \approx 0.021 \mathrm{~m}$ (filled square), $H_{\mathrm{s}} \approx 0.033$ $\mathrm{m}$ (filled diamond)

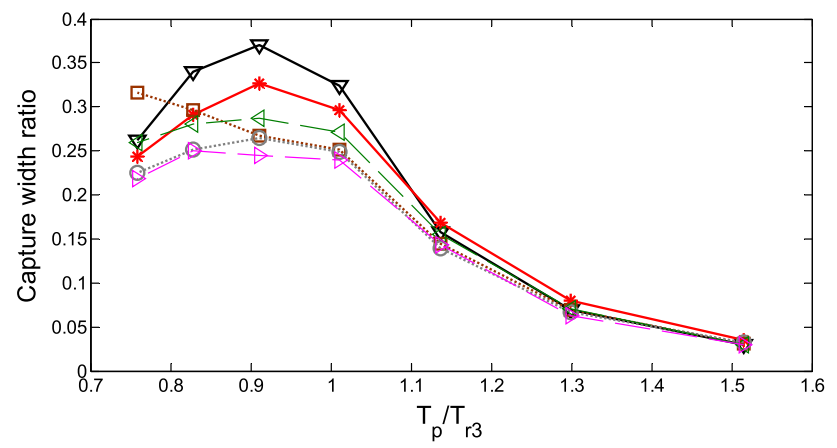

Fig. 6 Variation of capture width ratio (based on $T_{\mathrm{e}}$ ) with ratio of peak period $T_{\mathrm{p}}$ to resonant heave period on stern float $T_{\mathrm{r} 3}$ (based on WAMIT added mass) with $\gamma=3.3$ and cosine-based spreading function $s=\infty$ (uni-directional), 30 and 5 for rounded base floats. $s=\infty H_{\mathrm{s}} \approx 0.027$ $\mathrm{m}$ (asterisk), $H_{\mathrm{s}} \approx 0.042 \mathrm{~m}($ down triangle $) ; s=30 H_{\mathrm{S}} \approx 0.022 \mathrm{~m}$ (left triangle), $H_{\mathrm{s}} \approx 0.034 \mathrm{~m}$ (right triangle); $s=5 H_{\mathrm{s}} \approx 0.018 \mathrm{~m}$ (open square), $H_{\mathrm{s}} \approx 0.027 \mathrm{~m}$ (open circle)

Surprisingly with $\gamma=1.0$ as shown in Fig. 7, capture width ratio increases as directional spreading increases $(s$ reduces) and again magnitudes are larger for smaller $H_{\mathrm{s}}$; the values for $s=5$ and 30 are very similar. For both $\gamma$ values, the capture width ratio is greater than 0.2 across a broad range of periods.

\section{Results at larger scale with flat base floats}

Experiments at five times larger scale were undertaken in the University of Plymouth wave basin using floats with flat bases, shown in Fig. 8. Note the radius of curvature of the rounded base corners was $10 \mathrm{~cm}$, double the relative radius of the smaller scale. The wavemakers were again from Edinburgh Designs driven by the OCEAN software; the depth is $2.9 \mathrm{~m}$ and mass and inertia distributions are given in Table 5 . With a geometric scale factor of five applied, the mean

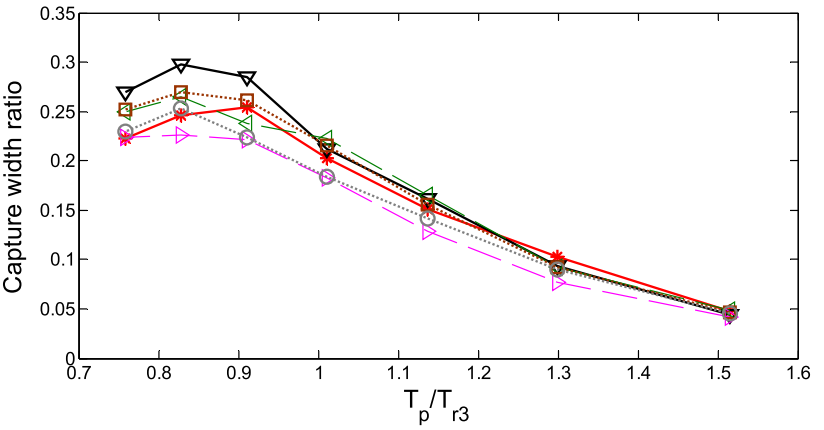

Fig. 7 Variation of capture width ratio (based on $T_{\mathrm{e}}$ ) with ratio of peak period $T_{\mathrm{p}}$ to resonant heave period on stern float $T_{\mathrm{r} 3}$ (based on WAMIT added mass) for $\gamma=1.0$ and spreading function $s=\infty$ (unidirectional), 30 and 5 for rounded base floats. $s=\infty H_{\mathrm{s}} \approx 0.021$ $\mathrm{m}$ (down triangle), $H_{\mathrm{s}} \approx 0.033 \mathrm{~m}$ (asterisk); $s=30 H_{\mathrm{s}} \approx 0.026 \mathrm{~m}$ (left triangle), $H_{\mathrm{s}} \approx 0.038 \mathrm{~m}$ (right triangle); $s=5 H_{\mathrm{s}}=0.021 \mathrm{~m}$ (open square), $H_{s} \approx 0.030 \mathrm{~m}$ (open circle)

damping factor $B_{\mathrm{d}}$ is scaled by $5^{4.5}=1397.5$. The values corresponding to the smaller scale of 1.4-2.3 Nms are in the range 2000-3200 Nms, while the available damper gives values in the range $2200-2850 \mathrm{Nms}$. There is thus overlap of the scaled values of the mean damping factor. Results of linear diffraction modelling with regular waves (Stansby et al. 2015) indicate that the maximum power capture at larger scale is achieved by increasing $B_{\mathrm{d}}$ by a factor of between 2 and 4 increasing power capture by $24 \%$. Since $B_{\mathrm{d}}$ values do not exactly scale the comparison between scales can only be approximate.

In the experiments with regular waves, the variation of average power output is again close to proportional to $H^{2}$ for different periods. Best-fit lines pass very close to the origin indicating negligible friction losses in the bearings.

For the flat base floats, Fig. 9 shows that the variation in capture width ratio is very similar at small and large scale with $\gamma=3.3$ for the smaller equivalent $H_{\mathrm{s}}$ values. For the larger equivalent $H_{\mathrm{s}}$ values, the capture width ratio is somewhat smaller at small scale. At the larger scale, curves are quite similar for $\gamma=1.0$ and 3.3.

An example of time variation of moment $M_{\mathrm{d}}$, angular displacement $\theta$ and power output is shown in Fig. 10 for $H_{\mathrm{s}}=0.23 \mathrm{~m}, T_{\mathrm{p}}=2.8 \mathrm{~s}$ and $\gamma=3.3$. The $M_{\mathrm{d}}$ time variation is again fitted to a model defined as $M_{f i t}=B_{0}+B_{d} \theta^{\prime \prime}$. The least squares goodness-of-fit $R^{2}$ is 0.94 in this case; the $B_{\mathrm{d}}$ value was $2536 \mathrm{Nms}$.

Capture width ratio from tests at both scales with directional spreading $s=30$ are shown in Fig. 11 for $\gamma=3.3$ and in Fig. 12 for $s=5$. It can be seen that values with equivalent larger $H_{\mathrm{s}}$ are somewhat larger at the larger scale with $s=30$ although quite similar with $s=5$. The values for the smaller wave height, tested at smaller geometric scale only, are generally slightly larger. 


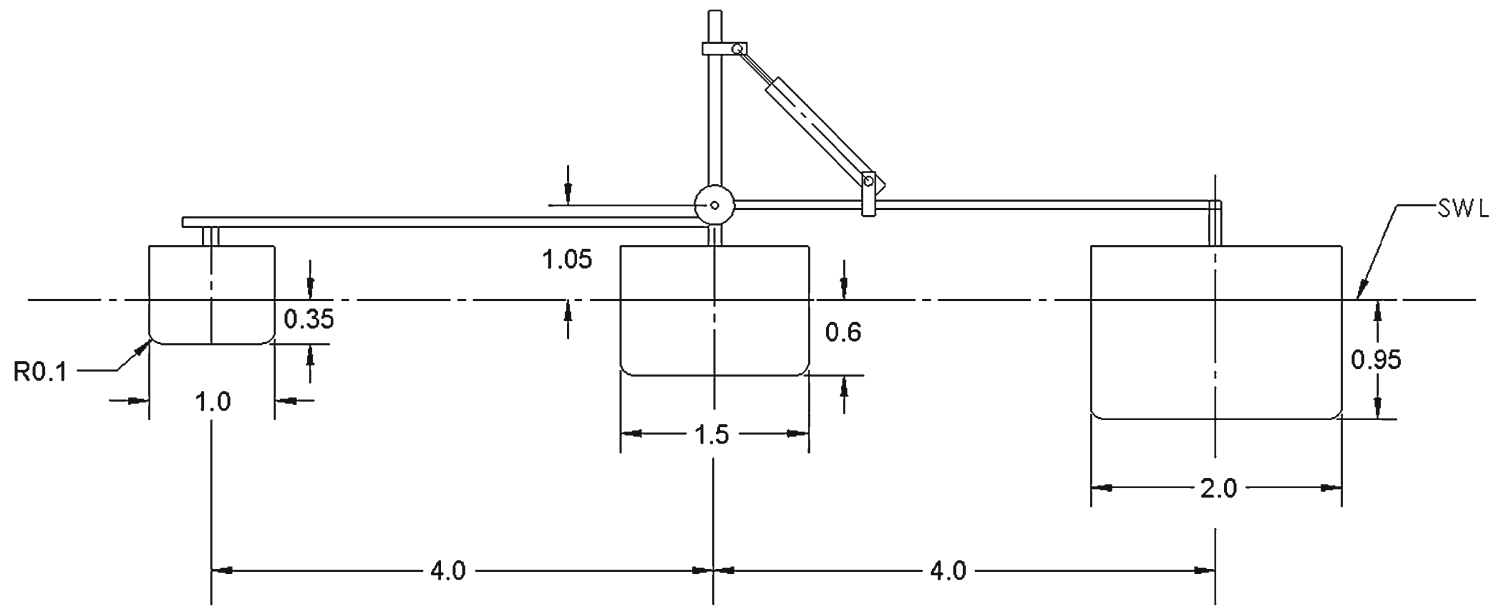

Fig. 8 Sketch of configuration at larger scale and dimensions (m)

Table 5 Mass distributions and scale with origin through bow float centreline and base of stern float inertias for five times larger

\begin{tabular}{llll}
\hline & Mass $(\mathrm{kg})$ & $x_{G}(\mathrm{~m})$ & $z_{G}(\mathrm{~m})$ \\
\hline Float 1 & 221 & 0 & 1.037 \\
Beam float 1 to 2 & 129 & 1.809 & 1.425 \\
Float 2 & 633 & 3.933 & 1.052 \\
Beam float 2 to 3 & 171 & 5.644 & 1.892 \\
Float 3 & 1137 & 7.546 & 0.745 \\
Actuator & 25 & 4.560 & 2.150 \\
Ballast float 2 & 225 & 4.00 & 0.355 \\
Ballast float 3 & 1725 & 8.00 & 0.050 \\
Combined floats 1 and 2 & 1223 & 3.05 & 0.954 \\
Combined float 3 & 3083 & 7.674 & 0.420 \\
Inertia about hinge floats 1 and 2 combined & $4467 \mathrm{~kg} \mathrm{~m}^{2}$ & & \\
Inertia about hinge float 3 & $53,005 \mathrm{~kg} \mathrm{~m}^{2}$ & & \\
\hline
\end{tabular}

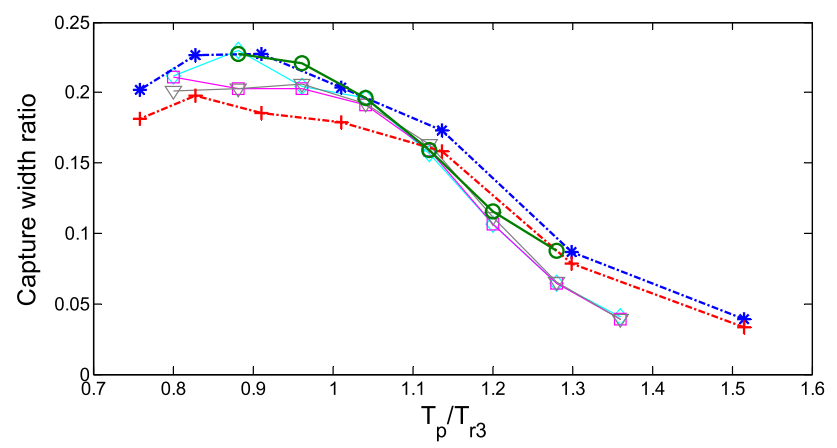

Fig. 9 Variation of capture width ratio (based on $T_{\mathrm{e}}$ ) with peak period $T_{\mathrm{p}}$ normalised by resonant stern float period $T_{\mathrm{r} 3}$ (with added mass from WAMIT) for different $H_{\mathrm{S}}$ at small and larger scale for unidirectional waves for flat-based floats. Large scale $H_{\mathrm{s}} \approx 0.14 \mathrm{~m}$ (open diamond), $H_{\mathrm{s}} \approx 0.19 \mathrm{~m}$ (open square), $H_{\mathrm{s}} \approx 0.25 \mathrm{~m}$ (open down triangle) with $\gamma=3.3$ Small scale $H_{\mathrm{s}} \approx 0.027 \mathrm{~m}$ (asterisk), $H_{\mathrm{s}} \approx 0.040 \mathrm{~m}$ (plus) with $\gamma=3.3$ Large scale $H_{\mathrm{s}} \approx 0.19 \mathrm{~m}$ (open circle) with $\gamma=1$

\section{Discussion}

Experimental analysis is undertaken with geometrically scaled models for which time and force scales are based on Froude scaling. The scale of the systems studied differs by a factor of five. The moments generated by the mechanical actuators were fitted reasonably by terms proportional to angular velocity and acceleration and a mean term, enabling the damping factor to be determined. The smaller-scale pneumatic actuator gives a wider range of equivalent damping factor values than the larger-scale hydraulic actuator. Comparisons between these experiments may therefore only be approximate. Damping factors at both scales are suboptimal based on prior analysis in regular waves for the flat base floats at the larger scale which implies that capture widths may be $24 \%$ larger. However, results in regular waves do not 

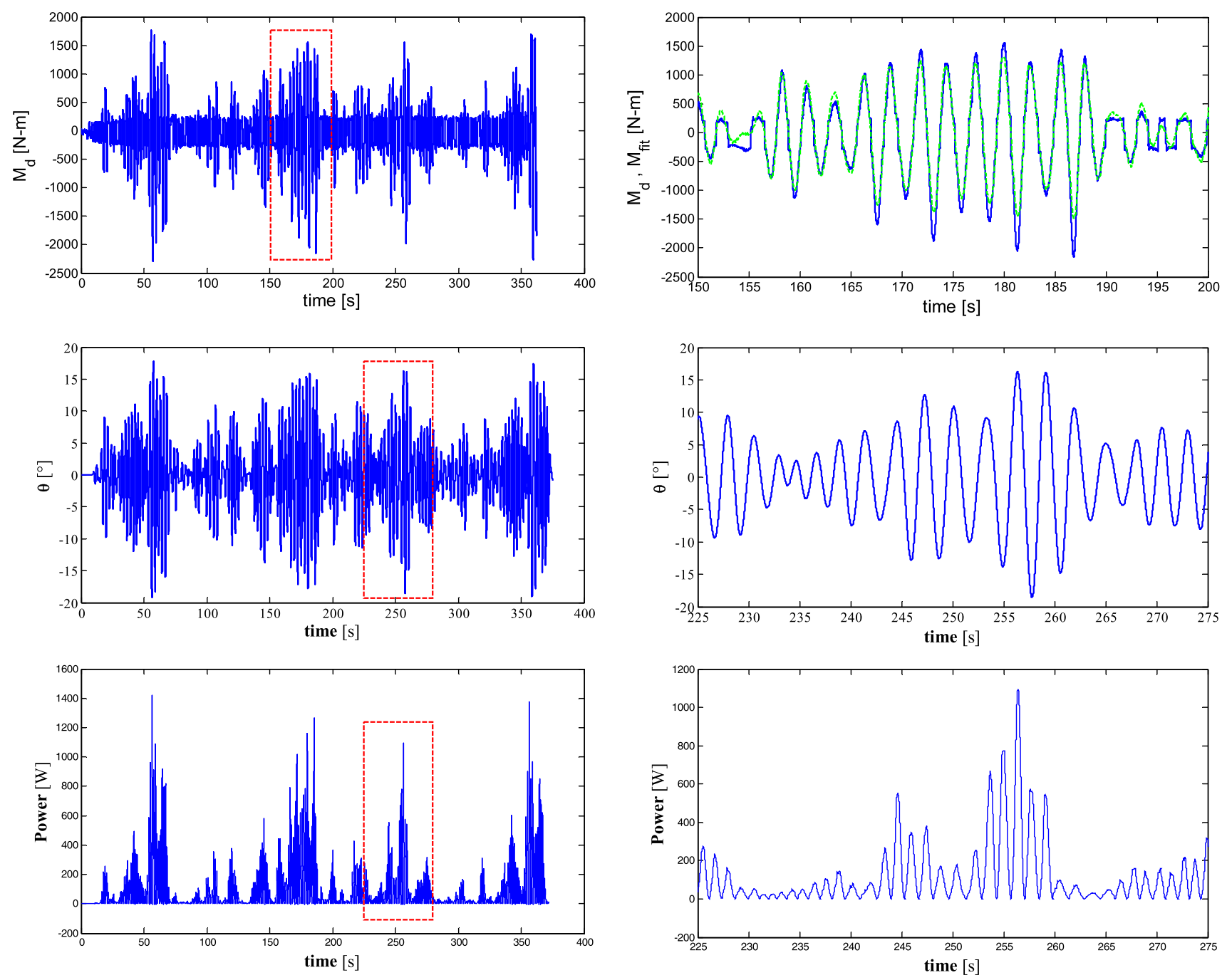

Fig. 10 Variation of moment, angle, output power and mechanical damping factor with time for $H_{\mathrm{s}}=0.23 \mathrm{~m}, T_{\mathrm{p}}=2.8 \mathrm{~s}$ and $\gamma=3.3$. The right column shows in detail the interval shown in the red box in the left column. For $M_{\mathrm{d}}$ the curve fit is shown by the dashed line on the right hand side

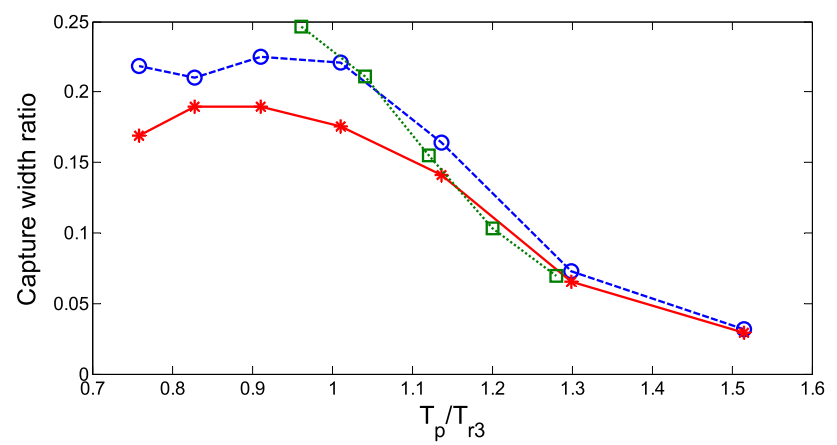

Fig. 11 Variation of capture width ratio (based on $T_{\mathrm{e}}$ ) with peak period $T_{\mathrm{p}}$ normalised by resonant stern float period $T_{\mathrm{r} 3}$ (with added mass from WAMIT) at small and large scale with $\gamma=3.3$ and spreading parameter $s=30$, for flat-based floats. Large scale $H_{\mathrm{s}} \approx 0.19 \mathrm{~m}$ (open square); small scale $H_{\mathrm{s}} \approx 0.027 \mathrm{~m}$ (open circle), $H_{\mathrm{s}} \approx 0.040 \mathrm{~m}$ (asterisk)

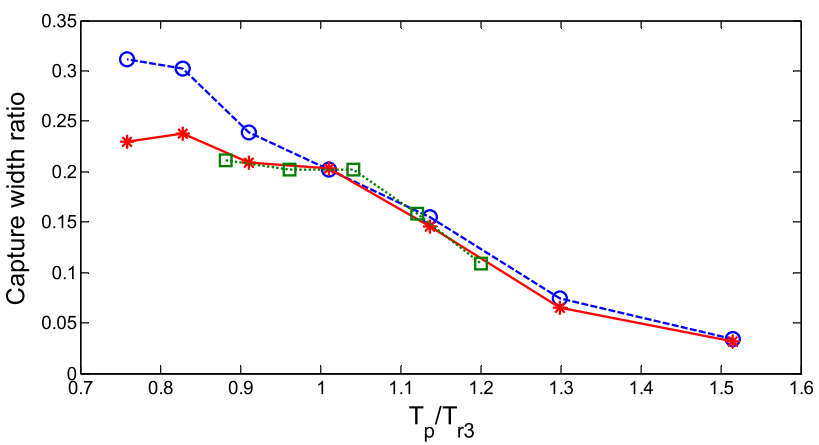

Fig. 12 Variation of capture width ratio (based on $T_{\mathrm{e}}$ ) with peak period $T_{\mathrm{p}}$ normalised by resonant stern float period $T_{\mathrm{r} 3}$ (with added mass from WAMIT) at small and large scale with $\gamma=3.3$ and spreading parameter $s=5$, for flat-based floats. Large scale $H_{\mathrm{s}} \approx 0.19 \mathrm{~m}$ (open square); small scale $H_{\mathrm{s}} \approx 0.017 \mathrm{~m}$ (open circle), $H_{\mathrm{s}} \approx 0.027 \mathrm{~m}$ (asterisk) 
necessarily transfer linearly to irregular waves or to floats of different shapes. Control of mechanical damping is of course desirable to enable more precise matching between scales as well as optimisation, although this was not possible in these tests and is difficult at small scale. Nevertheless, it is notable that qualitative similarity has been observed using quite different damping actuators.

The instantaneous power was measured without approximation providing accurate average power estimates. The experiments are thus valuable for exploring the power conversion potential of this system with novel features intended to give a marked improvement in capture width over existing configurations.

The size of the stern float defines the dimensions of the rest of the system and the diameter is specified as approximately $20 \%$ of the wavelength associated with a prominent wave period, so that forcing is predominantly inertial with minimal diffraction. The natural heave period of the stern float is designed to correspond approximately with the peak spectral period by specifying ballast and hence draft. The spacing between floats is set at about half the corresponding wavelength. The mid float is intended to have a somewhat lower natural period to correspond with lower wave periods (about $20 \%$ lower in this case) and the diameter is chosen to maximise mean power. This analysis was based simply on two heaving floats giving a diameter of three-quarters of the stern float diameter. Power is only slightly dependent on diameter for larger values. Clearly, a smaller diameter would also be advantageous for minimising fabrication material at full scale. The bow float was originally intended to provide a passive mooring point with sufficient buoyancy to support the beam to mid float. The larger of the two diameters tested was chosen to give more buoyancy without loss of overall power capture and it was not used as a mooring buoy, since it has significant motion contributing to power generation. The change in base shape on mid and stern float from flat to hemispherical and rounded with the same radius of curvature, respectively, gave a dramatic improvement in capture width emphasising the importance of drag minimisation.

The maximum capture width of over $35 \%$ wavelength with rounded bases in irregular waves and importantly over $20 \%$ over a broad range of peak periods for all values of spectral peakedness $(\gamma)$ and directional spreading(s) tested may be considered high values. The geometric configuration could probably be optimised further; a larger stern diameter should be considered which will influence the optimum diameter of the mid and possibly the bow float. In determining these natural heave periods, it was found that viscosity affects added mass as well as determining drag. The natural period of the stern float with the flat base in isolation was measured to be $1.12 \mathrm{~s}$ in a free decay test, while that calculated with added mass from diffraction analysis was $1.07 \mathrm{~s}$, a difference of $4.6 \%$ implying that $20 \%$ of added mass may be associated with viscous effects. Note that the mid and bow floats could not be tested in isolation due to lack of stability.

In irregular waves there is a flat peak in the variation of capture width with $T_{\mathrm{p}}$ with a maximum just below the heave resonant period of the stern float based on diffraction added mass. The peak is thus closer to the natural period accounting for viscous effects. That the capture width only decreases slightly for smaller $T_{\mathrm{p}}$ is probably due to the lower resonant period of the mid float and it would be useful to assess optimisation of this draft further to maximise overall energy capture in irregular waves for a representative wave climate.

Reducing spectral peakedness $\gamma$ from 3.3 to 1.0 reduces capture width with the rounded base floats, but has little effect with the flat base floats. Smaller $H_{\mathrm{s}}$ values generally give slightly higher capture widths indicating smaller drag effects. Increasing directional spread (reducing s) reduces capture width with $\gamma=3.3$, although there is little difference between $s=30$ and $s=5$. Perhaps, surprisingly directional spread results in slightly larger capture width with $\gamma=1$.

Results have not been represented for regular waves because of uncertainties in capture width associated with reflections and occasionally cross-basin seiching, although this was not observed at larger scale and reflection was a small effect; results are reported elsewhere (Stansby et al. 2015). This is a recognised limitation associated with tank testing, particularly for multi-body systems. For small arrays of heaving floats at similar scale, the response amplitude was obtained from the initial cycles only (Weller et al. 2010).

Mathematical modelling has not been applied in this paper which is a purely experimental investigation in irregular waves. Several different modelling approaches are appropriate. Modelling based on linear diffraction coefficients for added mass, radiation damping and excitation for all relevant modes will provide an efficient approach in the frequency or time domain, but an effective drag coefficient dependent on wave height and possibly period is needed to calibrate against experiment. The effect of viscosity on added mass also needs calibrating and there may be an effect on radiation damping. This modelling is thus a valuable adjunct to experiments for fast optimisation, but is of limited value in isolation. Viscous effects are included in full Navier-Stokes solvers with a free surface, typically RANS solvers in the volume of fluid (VOF) method. Such methods with a complex fluid mesh are exceptionally demanding computationally and subtle effects of boundary layer separation with turbulence which affect drag are very difficult to predict accurately. Nevertheless, further investigation through this type of modelling would be beneficial.

While further geometric optimisation is desirable, the optimisation and design of the power take-off (PTO) is expected to be of greater importance. This is actually very difficult to achieve physically at small scale and here a calibrated mathematical model is desirable to couple with algorithms 
for hydraulic control (based on proven hydraulic systems) to enhance and smooth power output.

For an offshore site with a known wave climate, the characterisation of the system in terms of capture width enables annual energy yield to be readily determined. While we are concerned with capture width, the device cost per MW capacity and levelised cost of energy determine practical viability. A simple preliminary economic analysis (Stansby et al. 2014) indicates that capital costs could be comparable to target costs for this stage of development given in Marine Energy Technology Roadmap (2010); this is competitive with offshore wind energy and without the associated environmental intrusion.

\section{Conclusions}

A three-float line absorber M4 has been proposed with float spacing of about half a wavelength to give anti-phase forcing between adjacent floats with excitation in heave, pitch and surge. The float size in terms of both diameter and draft increases from bow to stern, facilitating alignment of the line absorber with the wave direction. The heave resonance periods on mid and stern float are different to optimise power capture for a range of wave periods and the mid float diameter is sized to give maximum power output (based on an idealised heave analysis). Anti-phase surge forcing on mid and stern floats is significant, although without a hydrostatic restoring force for resonance. This forcing is exploited by the relative pitch about the hinge point well above deck level. The aim is that power from the different forms of excitation and forcing combines to give a markedly improved capture width over what has been achieved previously in wave energy conversion. This has been investigated at laboratory scales of about 1:40 and 1:8 in irregular waves with different spectral peakedness and directional spreading. Capture width is the important assessment criterion. In irregular waves, the capture width is above $20 \%$ of the wavelength associated with the energy period, for a configuration with rounded base floats, across a broad range of wave periods and with different combinations of both directional spreading and spectral peakedness typical of an offshore site (with appropriately scaled periods); the maximum was over $35 \%$. This may be considered large relative to existing systems. The tests indicate the effect of viscosity on both drag and added mass and indicate the importance of minimising drag for increasing power output. While some geometric optimisation has been undertaken, more would be beneficial. Most importantly, optimisation of the power take-off (PTO) control is required since only simple actuators are used in these reduced-scale experiments.

Acknowledgments For the larger device, we acknowledge support from Cammell Laird on fabrication and naval architecture analysis through D. Williams and from Rexroth Bosch for provision of the hydraulic actuator through L. Verdegem. The Plymouth COAST laboratory provided a high-quality facility and technical service. CONACYT provided the $\mathrm{PhD}$ studentship for the second author. The project is supported by EPSRC through the Supergen Marine Challenge 2 Grant Step-WEC (EP/K012487/1). The patent application was made by Peter Stansby of M4 WavePower Ltd (Stansby 2013). The reviewers have made a valuable contribution.

Open Access This article is distributed under the terms of the Creative Commons Attribution License which permits any use, distribution, and reproduction in any medium, provided the original author(s) and the source are credited.

\section{Appendix}

\section{Analytical two-body heave analysis in regular waves}

For two cylindrical bodies in regular linear waves oscillating in heave only, denoted by mode 3 on body 1 (bow) and mode 9 on body 2 (stern), $(\boldsymbol{M}+\boldsymbol{A}) \ddot{z}=-\left(\boldsymbol{B}+\boldsymbol{B}_{d}\right) \dot{z}-\frac{1}{2} \rho S C_{D} \dot{z}|\dot{z}|-$ $\rho g S z+\boldsymbol{F}$, where mass $\mathbf{M}=\left[\begin{array}{ll}M_{1} & 0 \\ 0 & M_{2}\end{array}\right]$, added mass $\mathbf{A}=$ $\left[\begin{array}{ll}A_{33} & A_{39} \\ A_{93} & A_{99}\end{array}\right]$, radiation damping $\mathbf{B}=\left[\begin{array}{ll}B_{33} & B_{39} \\ B_{93} & B_{99}\end{array}\right]$, vertical hydrodynamic excitation force $\mathbf{F}=\left[\begin{array}{l}F_{3} \\ F_{9}\end{array}\right]$, and $F_{3}$ accounts for effect of body 2 on body 1 and $F_{9}$ accounts for effect of body 1 on body 2 . $\rho g S$ is sometimes known as hydrodynamic stiffness where $S$ is cross-sectional area at the water plane. The coefficients and forces were obtained from WAMIT. The mechanical damping with coefficient $B_{\mathrm{d}}$ is due to relative vertical velocity between bodies, and adding the effect of linearised drag due to vertical float velocity with drag coefficient $C_{\mathrm{D}}$ gives $\mathbf{B}_{\mathbf{d d}}=\left[\begin{array}{ll}-B_{d}-S_{1} K \dot{z}_{1_{0}} & B_{d} \\ B_{d} & -B_{d}-S_{2} K \dot{z}_{2_{0}}\end{array}\right]$, where suffix 0 implies amplitude and $K=\frac{1}{2} \rho C_{D} 3 \pi / 8 \sqrt{2}$. The complex velocity amplitude $\dot{z}_{0}=\frac{\mathbf{F}_{0}}{\left[\mathbf{B}+\mathbf{B}_{\mathbf{d d}}+\omega\left(\mathbf{M}+\mathbf{A}-\frac{\rho \mathrm{gS}}{\omega^{2}}\right) \mathbf{i}\right]}$, where $\mathbf{F}_{0}$ is the complex force amplitude and $\omega$ is the angular wave frequency. Finally, the instantaneous power out $P_{\text {out }}=$ $B_{d}\left(\dot{z}_{2}-\dot{z}_{1}\right)^{2}$ and average power $P_{a v}=0.5 B_{d}\left|\dot{z}_{2_{0}}-\dot{z}_{1_{0}}\right|^{2}$, where $\dot{z}_{2_{0}}, \dot{z}_{1_{0}}$ are complex amplitudes. This equation set was solved iteratively using Matlab, with the initial estimates for float velocity in the drag term set to zero, to give average power. A representative $C_{\mathrm{D}}=1.8$ was used following comparisons of linearised modelling with three body experiments (as used here) in Stansby et al. (2015).

\section{References}

Babarit A, Clément AH (2006) Optimal latching control for a wave energy device in regular and irregular waves. Appl Ocean Res 28:77-91 
Borgman LE (1969) Directional spectral models for design use. In: Proceedings of Offshore Tech. Conf., Houston, Paper No. OTC 1069, pp 721-746

Caljouw R, Harrowfield D, Mann LD, Fievez J (2011) Testing and model evaluation of a scale CETO unit. Towards the deployment of a commercial scale CETO Wave Energy Converter. In: Proceedings of the 9th European Wave and Tidal Energy Conference, Southampton

Cameron L, Doherty R, Henry A, Doherty K, Van't Hoff J, Kay D et al (2010) Design of the next generation of Oyster wave energy converter. In: Proceedings of the 3rd International Conference on Ocean Energy, Bilbao, Spain pp 1-12. Available from http://www. icoe-conference/library/conference/icoe_2010/

Cruz J (2008) Ocean wave energy. Springer, New York

Drew B, Plummer AR, Sahinkaya MM (2009) A review of wave energy converter technology. In: Proceedings of the IMechE vol 223 Part A: J Power and Energy, pp 887-902

de Falcão FO (2010) Wave energy utilization: a review of the technologies. Renew Sustain Energy Rev 14:899-918

Falnes J (2002) Ocean waves and oscillating systems. Cambridge University Press, Cambridge

Farley FJM, Rainey RCT, Chaplin JR (2012) Rubber tubes in the sea. Phil Trans Roy Soc A 370:381-402

Hansen RH, Kramer MM (2011) Modelling and control of the wavestar prototype. In: Proceedings of the 9th European Wave and Tidal Energy Conference, Southampton, UK

Lavelle J, Kofoed JP (2011) Experimental testing of the Langlee wave energy converter. In: Proceedings of the 9th European Wave and Tidal Energy Conference, Southampton, UK

Lok K, Stallard T, Stansby P, Jenkins N (2014) Optimisation of a clutchrectified power take off system for a heaving wave energy device in irregular waves with experimental comparison. J Marine Energy $8: 1-16$

Longuet-Higgins MS, Cartwight DE, Smith ND (1963) Observations of the directional spectra of sea waves using the motions of a floating buoy. Ocean Wave Spectra, Prentice_Hall, pp 111-136

Marine Energy Technology Roadmap (2010) Energy Technologies Institute and UK Energy Research Centre, University of Edinburgh
Masuda Y, Kimura H, Gao X, Mogensen RM, Andersen T (1995) Regarding BBDB wave power generation plant. In: Proceedings of the 2nd Wave Energy Power Conference, pp 69-76

McCabe AP, Bradshaw A, Meadowcroft JAC, Aggidis G (2006) Developments in the design of the PS Frog Mk 5 wave energy converter. Renew Energy 31(2):141-151

Mei CC (1989) The applied dynamics of ocean surface waves. World Scientific Publishing, Singapore

Ocean energy. Available online http://www.oceanenergy.ie. Accessed 7 Dec, 2014

Polinder H, Damen MEC, Gardner F, Linear PM (2004) Generator system for wave energy conversion in the AWS energy conversion. IEEE Trans Energy Convers 19(3):583-589

Rogers D, Bolton King G (1997) Wave generation using Ocean and Wave, version 3.62

Stallard TJ, Weller SD, Stansby PK (2009) Limiting heave response of a wave energy device by draft adjustment with upper surface. Appl Ocean Res 31(4):282-289

Stansby P, Carpintero Moreno E, Stallard T, Maggi A (2015) Threefloat broad-band resonant line absorber with surge for wave energy conversion. Renew Energy 78:132-140

Stansby P, Carpintero Moreno E, Stallard T, Maggi A, Eatock Taylor R (2014) Wave energy conversion with high capture width by the three-float line absorber M4. In: Proceedings Renewable Energies Offshore, Lisbon

Stansby P (2013) Surge based wave energy converter PCT/GB2013/ 050787

WAMIT (2004) User manual versions 6.2, 6.2PC, 6.2S. 6.2SPC.WAMIT Inc. http://www.wamit.com

Weller SD, Stallard TJ, Stansby PK (2010) Response of a small array due to irregular waves: comparisons to predictions based on measured irregular wave response. In: Proceedings of the 20th Conference of International Society of Offshore and Polar Engineering (ISOPE), Beijing, China, 20-25 Jun 2010. pp 900-907

Yemm R, Pizer D, Retzler C, Henderson R (2012) Pelamis: experience from concept to connection. Phil Trans Roy Soc A 370(1959):365380 